\title{
Estrategias bioclimáticas para clima frío tropical de altura. Validación de prototipo en Orduña, Puno, Perú.
}

\author{
Bioclimatic strategies for high altitude tropical cold climate. \\ Prototype validation in Orduña, Puno, Perú.
}

\section{Resumen}

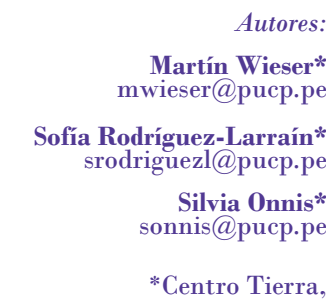

Departamento de Arquitectura Pontificia Universidad Católica del

Perú, PUCP

Perú

Recibido: 20/Mar/2020 Aceptado: 09/Nov/2020
L

as condiciones de frío extremo en la región del altiplano peruano generan problemas recurrentes que afectan la salud y la economía de la población local. El limitado desempeño térmico de las viviendas sobre los 4200 m.s.n.m., sumado a una economía de subsistencia y a la necesaria dispersión en el territorio debido a su actividad ganadera, agrava la situación de vulnerabilidad de la población. En el marco de un proyecto de transferencia tecnológica para la mejora térmica y constructiva de la vivienda altoandina, se diseñó, construyó y validó, junto con la población local, un prototipo bioclimático y sismorresistente en la localidad de Orduña, Puno, Perú. El presente documento se centra en las estrategias bioclimáticas pasivas implementadas en el prototipo, lográndose demostrar que es posible obtener confort térmico en dichas condiciones extremas a partir de la utilización casi exclusiva de materiales locales y naturales.

Palabras clave: aislamiento térmico; arquitectura bioclimática; confort térmico; heladas; vivienda altoandina.

\section{Abstract:}

Extreme cold conditions in the Peruvian highland region generate recurring problems that affect the health and economy of the local population. The limited thermal performance of the dwellings above 4200 meters above sea level, added to a subsistence economy and the necessary dispersion in the territory, due to their livestock activity, aggravates the situation of vulnerability of the population. Within the framework of a technology transfer project for the thermal and constructive improvement of the high Andean house, a bioclimatic and seismic resistant prototype in the town of Orduña, Puno, Peru, was designed, built and validated, together with the local population. This document focuses on the passive bioclimatic strategies implemented in the prototype, demonstrating that it is possible to obtain thermal comfort in these extreme conditions from the almost exclusive use of local and natural materials.

Keywords: thermal insulation; bioclimatic architecture; thermal comfort; frost; High Andean house. 


\section{Introducción}

La zona altoandina del sur del Perú tiene un clima extremo en el que coexisten temperaturas muy bajas de noche frente a temperaturas moderadas y radiación solar extremadamente alta durante el día. Sobre los 4200 m s.n.m., principalmente en los meses de invierno, es frecuente el fenómeno meteorológico de las heladas, con temperaturas nocturnas por debajo del punto de congelación. Este fenómeno dificulta el confort térmico y la salud de los habitantes de estas regiones, incluso poniendo en riesgo sus vidas, principalmente las de niños y ancianos. En esta zona habitada hasta los 4800 m s.n.m., que corresponde a la región natural Puna (Pulgar Vidal, 1996), la rigurosidad climática y la composición de los suelos no permiten la agricultura, y la actividad económica se limita casi exclusivamente a la crianza de camélidos sudamericanos.

Las familias se asientan de manera dispersa en el territorio y cuentan con una economía de subsistencia que limita la inversión en los edificios que habitan, también denominados 'cabañas'. La recurrencia de fenómenos meteorológicos adversos, como las heladas y las sequías, además de la introducción inadecuada de nuevos materiales en los procesos constructivos locales, como la plancha ondulada metálica y el vidrio, complican seriamente las posibilidades de desarrollo de estas comunidades. Las condiciones de confort al interior de las cabañas son extremadamente pobres, inhabitables desde el punto de vista térmico, con temperaturas interiores nocturnas que suelen rondar siempre $\operatorname{los} 0{ }^{\circ} \mathrm{C}$.

Bajo esta premisa se desarrolló un proyecto de transferencia tecnológica, financiada en una primera etapa por el Consejo Nacional de Ciencia y Tecnología e Innovación Tecnológica (CONCYTEC) y la Pontificia Universidad Católica del Perú (PUCP), cuyo objetivo fue el de investigar el hábitat rural a través del intercambio de conocimientos para favorecer el desarrollo local en la zona altoandina (Rodríguez-Larraín, Onnis y Vargas-Neumann, 2016). En una primera etapa del proyecto, se construyó un prototipo bajo la perspectiva de la prevención de riesgo de desastres a través de una arquitectura bioclimática y sismorresistente. Se trabajó específicamente en la comunidad de Orduña, ubicada en el distrito de Santa Lucía y provincia de Lampa, departamento de Puno.

Esta primera etapa del proyecto se desarrolló entre 2014 y 2016 y consideró las fases de diagnóstico del hábitat, intercambio de conocimientos, diseño participativo, elaboración y validación de la propuesta y construcción de la vivienda piloto o prototipo. Terminada la construcción, que incluyó la capacitación de promotores técnicos (Onnis, Rodríguez-Larraín, Meli y Loayza León,
2018), se efectuó el monitoreo del desempeño térmico y constructivo. Resumiendo las estrategias bioclimáticas consideradas desde la primera etapa, estas fueron: el aislamiento de la envolvente a partir del uso de materiales naturales, la hermeticidad y la ganancia solar.

Los resultados, desde el punto de vista estrictamente térmico en esta primera etapa, fueron alentadores pero insuficientes (Jiménez, Wieser y Biondi, 2017). El desempeño térmico, si bien fue mejor que el de las cabañas típicas, estuvo por debajo de las condiciones mínimas esperadas de confort y de las propias expectativas del equipo de investigación.

Se identificaron posibilidades específicas de mejora del desempeño que fueron plasmadas en una segunda etapa: ampliación de las dimensiones del sistema de captación solar, incremento del aislamiento de la cubierta, aumento de la hermeticidad en los vanos y la adecuada manipulación de las aberturas. Ejecutadas las mejoras, en esta segunda etapa se realizaron nuevamente mediciones de desempeño térmico y de calidad del aire.

El presente documento repasa las estrategias utilizadas y los resultados térmicos obtenidos desde el inicio de proyecto y se centra en aquellos registrados a partir de la segunda intervención. Previamente se caracteriza con mayor detalle el ámbito geográfico y climático de la zona, se identifica la zona de confort y se describen las condiciones actuales de las viviendas locales.

\section{Consideraciones previas}

\section{1. Ámbito geográfico y climático}

La comunidad de Orduña la conforman alrededor de 50 familias. Se ubica a 4670 m s.n.m., a una latitud de $15^{\circ} 25^{\prime} 55^{\prime \prime}$ Sur y una longitud de $70^{\circ} 53^{\prime} 14^{\prime \prime}$ Oeste (Figura 1). El clima altiplánico es riguroso y el paisaje es muy variado según la época del año debido al régimen marcadamente estacional de las lluvias y de las eventuales nevadas y granizadas.

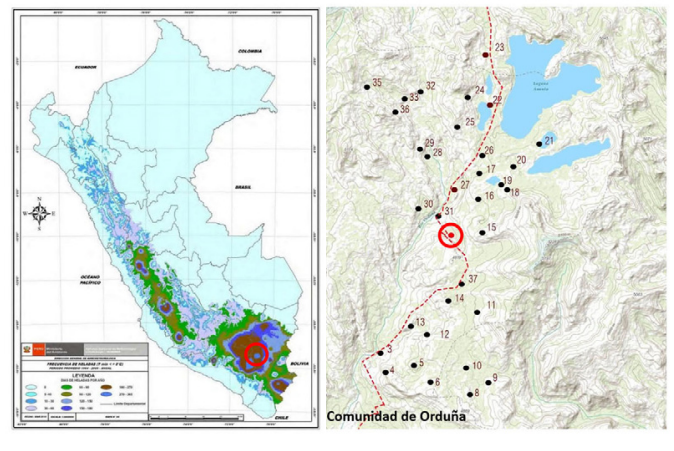

Figura 1: Ubicación de la localidad de Orduña sobre mapa de recurrencia de heladas en el Perú

Fuente: Ministerio del Ambiente (2019) y Centro Tierra, PUCP (2018)

. 
Las familias de la comunidad cuentan con una pequeña vivienda-depósito en el centro poblado con servicios de agua y electricidad, pero la utilizan solo para pernoctar una o dos noches cada dos semanas, coincidiendo con los días de feria. Dichas viviendas, generalmente de adobe, tienen dormitorios y ambientes que sirven de almacén (Figura 2, arriba). Las familias cuentan, a su vez, con una vivienda asociada a su actividad económica casi exclusiva, como es el pastoreo de alpacas. Estas viviendas, denominadas cabañas (Figura 2, abajo), se encuentran aisladas en zonas de mayor altitud aún, alejadas del centro poblado hasta en $10 \mathrm{~km}$ y separadas entre ellas generalmente entre 1 y $2 \mathrm{~km}$. Son en estas cabañas en las que se reside de manera casi continua, al estar asociadas a la actividad de pastoreo. Los animales pasan la noche en corrales anexos delimitados únicamente con cercos de piedra; en el caso de estar parcialmente techados, se les denomina cobertizos. Cabe mencionar que las familias suelen tener una vivienda adicional en alguna ciudad cercana, en la que residen ocasionalmente algunos miembros de las mismas por cuestiones de estudio, trabajo o salud, generalmente en las ciudades de Santa Lucía o Juliaca, ubicadas a unos 70 y 130 km de Orduña respectivamente.
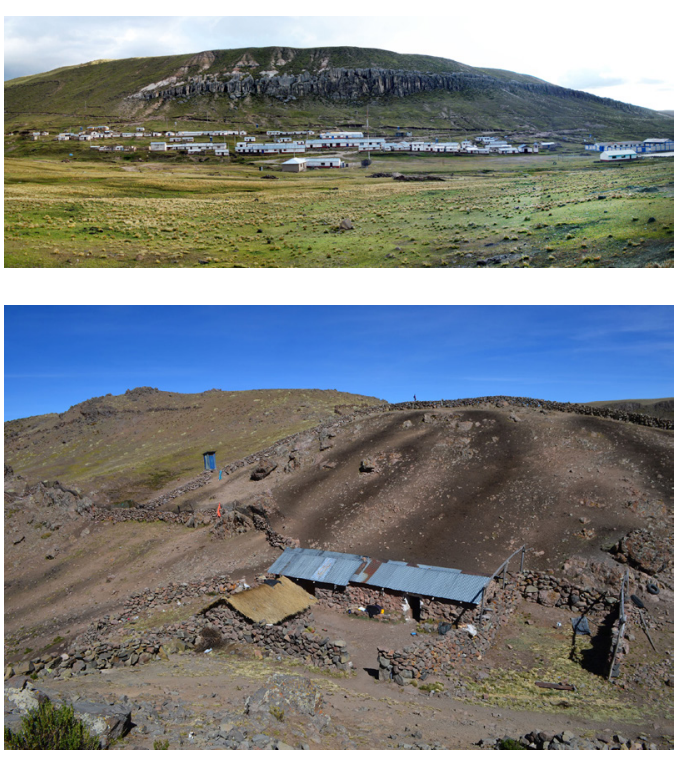

Figura 2: Vista panorámica de la Comunidad de Orduña y disposición típica de vivienda rural en los alrededores de poblado (Cabaña Tolane)

Fuente: Centro Tierra, PUCP (2018)

En la misma localidad de Orduña, no existe una estación meteorológica del Servicio Nacional de Meteorología e Hidrología (SENAMHI). La más cercana en la que se han registrado datos meteorológicos se ubica en la localidad de Pinaya ${ }^{1}, 16 \mathrm{~km}$ al Sur y 250 metros por debajo de la altitud de Orduña. Esta fuente oficial permitió tener una primera idea bastante cercana de las condiciones climáticas del emplazamiento. Una segunda fuente para la obtención de datos climáticos ha sido el software Meteonorm², el cual permitió la generación de un archivo epw, con el que se realizaron las simulaciones informáticas.
Una tercera fuente ha sido la recopilación de mediciones de datos in situ, mediante una estación meteorológica, realizada por el propio proyecto en diferentes momentos del año, desde noviembre de 2015 hasta enero de 2018. En términos generales, los resultados de las tres fuentes tienen coincidencias y permiten presentar los datos climáticos en las siguientes imágenes (Figura 3). Se presentan igualmente los datos de temperatura y humedad relativa del aire graficados sobre el ábaco psicrométrico (Figura 4).

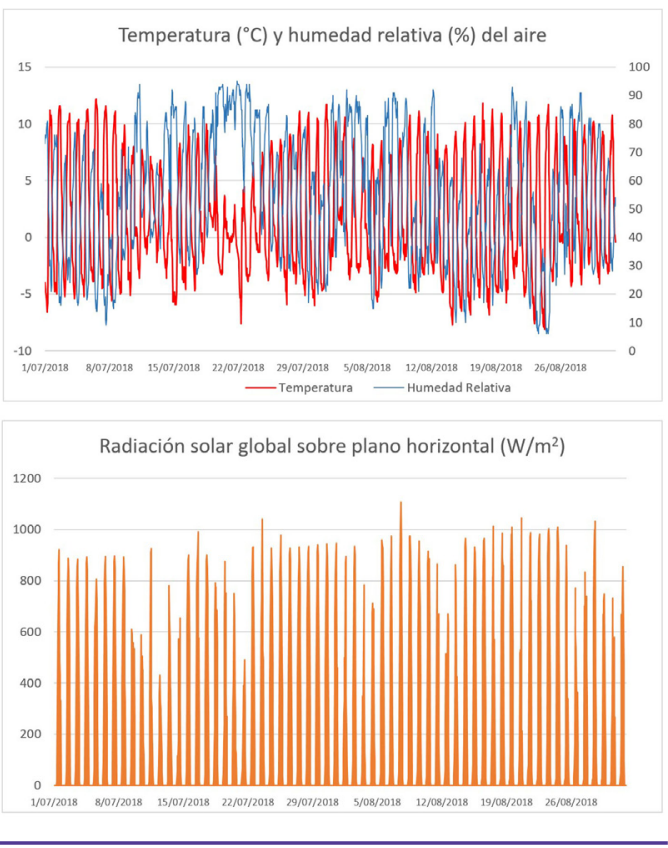

Figura 3: Temperatura y Humedad Relativa del aire (arriba) y radiación solar (abajo) en la localidad de Orduña Fuente: Elaboración propia a partir de mediciones in situ entre Julio y Agosto de 2018 (2018).

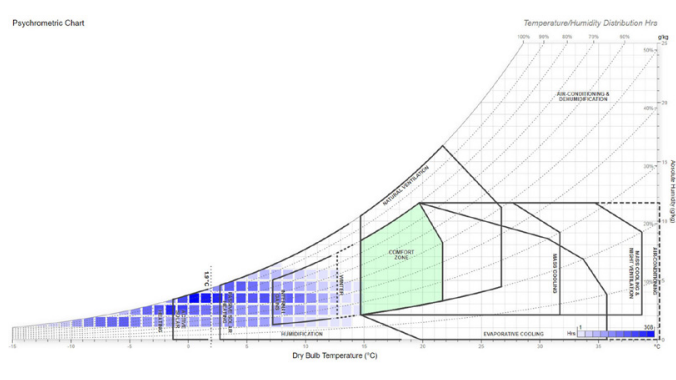

Figura 4: Ábaco psicrométrico con valores de temperatura y humedad relativa del aire de la localidad de Orduña

Fuente: Datos obtenidos de Meteonorm y graficados con el software 'Psychrometric Chart' desarrollado por Marsh, A. (2019). Se superpone la zona de confort y las estrategias de diseño sugeridas por Givoni (1998) y la temperatura exterior media de $1,93^{\circ} \mathrm{C}(2019)$

${ }^{1}$ Datos obtenidos de la Dirección de Redes de Observación y Datos de SENAMHI, entre los años de 1964 y 1969. https://www.senamhi.gob. pe/mapas/mapa-estaciones/_dat_esta_tipo.php?estaciones $=000773$ 2 Meteonorm Software es un programa informático desarrollado por Meteotest, para brindar y generar datos climáticos de cualquier lugar del mundo a partir de la interpolación de datos de estaciones cercanas. https://www.meteonorm.com/ 
El clima de la localidad de Orduña se puede resumir como un clima frío tropical de altura, cuyas principales características son la presencia de una alta oscilación térmica, con días frescos, ligeramente fríos y noches muy frías; durante las horas más frías de la madrugada, y a lo largo de todo el año, las temperaturas suelen estar siempre por debajo de $0^{\circ} \mathrm{C}$, generalmente entre $-4^{\circ} \mathrm{C}$ y $-8^{\circ} \mathrm{C}$. La diferencia estacional está marcada principalmente por el régimen de precipitaciones, que se dan generalmente en forma de granizo, las cuales son frecuentes en el verano (150 $\mathrm{mm}$ de promedio mensual), y casi inexistentes durante el invierno; cabe recordar que, al estar emplazada la localidad en el hemisferio sur, la temporada de invierno se da entre los meses de junio y septiembre. Durante el invierno, además de presentarse temperaturas ligeramente más bajas durante las noches, el cielo suele estar despejado y, por lo mismo, la presencia de radiación solar directa es prácticamente constante: alrededor de $1000 \mathrm{~W} / \mathrm{m}^{2}$ como valor típico al mediodía. Se compensa, de esta forma, la menor duración del día y la mayor inclinación del sol del invierno frente a la mayor nubosidad durante el verano, siendo finalmente la radiación global muy estable a lo largo de todo el año, generalmente entre 5 y $6 \mathrm{~kW} / \mathrm{m}^{2}$ acumulado diario sobre el plano horizontal.

\subsection{Condiciones actuales de las cabañas en Orduña}

A pesar de la incorporación paulatina de nuevos materiales en la construcción de las cabañas de la región, y de manera más evidente con la inclusión de la plancha ondulada metálica en los techos, la tipología formal y la configuración de los muros se ha mantenido prácticamente invariable. Dichos muros han sido resueltos tradicionalmente con piedras de tamaño mediano, las cuales se colocan unas sobre otras, y se utiliza el barro para tapar los intersticios. Al no ser piedras asentadas, su capacidad sismorresistente es muy limitada. Desde el punto de vista térmico, el muro tiene una alta inercia, pero a pesar del espesor considerable que se logra, su capacidad de aislamiento es muy limitada debido a la alta conductividad térmica del material, alrededor de 2,4 W/mK. Ocasionalmente se utiliza el adobe, pero las dificultades asociadas a su fabricación y secado en condiciones de temperaturas tan bajas desalientan su mayor utilización.

Las cubiertas han sido resueltas tradicionalmente con tejidos de ichu, fibra vegetal que conforma los pastizales y abunda en la región. El ichu es un buen aislante térmico y permite impermeabilizar el ambiente interior, al mismo tiempo que facilita un intercambio controlado de aire con el exterior. El ichu ha sido reemplazado paulatinamente por la plancha ondulada metálica en los techos; estas son generalmente de acero y zinc o de aluminio. E poco interés en continuar utilizando el ichu responde principalmente a que la plancha ondulada tiene un costo muy bajo y resulta muy fácil de manipular; ello, además de la gran dedicación de tiempo que requiere la confección de la cubierta de ichu y a la poca durabilidad que posee, ya que es necesario renovarla cada dos o tres años. El problema de la plancha metálica es que tiene una capacidad de aislamiento extremadamente pobre debido tanto a su espesor reducido como a su alta conductividad térmica, alrededor de $0,3 \mathrm{~mm}$ y 50 $\mathrm{W} / \mathrm{mK}$ respectivamente. Es cierto igualmente que, con la radiación impactando perpendicularmente sobre el techo, la calamina metálica permite elevar notoriamente la temperatura interior durante el día, pero dicha ganancia se pierde con rapidez en las primeras horas de la noche.

En cuanto a la disposición de las cabañas, estas están conformadas por un conjunto de volúmenes que se complementan hacia el exterior con muros de piedra relativamente bajos y una puerta, de tal forma que se crea un patio central protegido de los vientos (ver Figura 2); dicho patio se convierte en un espacio de uso intensivo por aquellos miembros de la familia que se quedan durante el día, generalmente cocinando y cuidando a los niños más pequeños. La eventual separación de los volúmenes no parece ser la mejor opción en un clima tan riguroso y de alta oscilación térmica, pero cobra sentido en la medida que generan los límites del patio y que muchos de ellos no suelen estar habitados; al ser depósitos o dormitorios de visitas eventuales, no son fuentes de calor que aportarían en caso de que se adosen unos a otros.

Entre otras características resaltantes de la arquitectura vernácula local, está la ausencia de vanos de ventana o, eventualmente, la presencia de aberturas muy pequeñas que, más que iluminar, permiten el control del ganado desde el interior. El vidrio no ha sido introducido aún de manera sistemática en la arquitectura, aunque últimamente se comienzan a incorporar elementos traslúcidos plásticos en las cubiertas, permitiendo el ingreso de la luz y del calor a ambientes tradicionalmente muy oscuros. En el caso de los muros, se pueden encontrar algunas situaciones en las que se ha reemplazado una o un grupo de piedras del muro por un elemento de vidrio simple o de plástico, unidos al muro con el mismo barro.

Las puertas son muy pequeñas $y$, en el caso de las estancias utilizadas para dormir, estas suelen orientarse hacia el Este, lo que permite el ingreso de radiación solar directa a través de ellas desde las primeras horas de la mañana. A partir de este momento, la temperatura del aire exterior sube abruptamente y alrededor del mediodía termina siendo notoriamente superior a la que hay en el interior, por lo que es muy usual dejar la puerta de dichas estancias abierta hasta media tarde. Anteriormente las puertas se cerraban durante la noche con pieles o telas, actualmente se suelen hacer de planchas metálicas o de madera.

En términos generales, la hermeticidad de los ambientes es muy pobre debido a los intersticios que existen en los vanos y en la unión entre el muro y el techo. Como características adicionales se pueden identificar que las alturas interiores de los ambientes son muy bajas, generalmente alrededor de 1,80 m entre el piso y el techo, y que el suelo interior es de tierra apisonada, lo que dificulta el control de la humedad, sobre todo en la época de lluvias.

\subsection{El confort térmico en el clima frío tropical de altura}

En una situación tan particular y extrema como la descrita, no resulta fácil identificar la condición al interior de una vivienda en la que se puede considerar que existe confort térmico. Los hábitos de uso de la vivienda en estas regiones altoandinas son igualmente singulares. 
El trabajo de pastoreo obliga a iniciar las actividades poco antes de las 5 de la madrugada, momento que coincide con las horas más frías, para salir con el ganado a los primeros indicios de luz natural. Los dormitorios se utilizan exclusivamente para pernoctar y dicha actividad la realizan con la misma ropa que se lleva puesta durante el día, a la que suman una gran cantidad de mantas, generalmente seis o más, para contrarrestar el frío al interior del ambiente.

Con todas estas particularidades, y al no haber estudios específicos sobre el confort térmico en regiones tan altas del altiplano, se tomará como una primera referencia el modelo de confort adaptativo para realizar una valoración provisional de las condiciones interiores requeridas. Dicho modelo permite identificar rangos de temperatura interior considerados como confortables, a partir de una correlación demostrada que existe entre la temperatura media exterior del emplazamiento y la de confort al interior de los edificios. Este se basa en la capacidad de adaptación del ser humano, a partir de diversos mecanismos, conscientes o inconscientes, para alcanzar una condición de confortabilidad: modificación en la generación de calor interno, cambios en la velocidad de pérdida de calor corporal, modificación del ambiente térmico o eligiendo un ambiente diferente. Como ya se adelantó, estos modelos permiten únicamente aproximaciones, ya que sus resultados pueden ser cuestionables cuando son confrontados con las respuestas de los usuarios en un clima extremo como el planteado y debido a las particularidades culturales de sus pobladores.

Según los modelos de confort adaptativo más reconocidos, como es el de Nicol y Humphreys (1998, 2002) y DeDear y Brager (1998), considerando las condiciones de temperatura exterior de los meses más fríos y asumiendo la condición de satisfacción del $80 \%$ de los usuarios $\left(+/-3,45^{\circ} \mathrm{C}\right.$ ) (DeDear y Brager, 1998), se presenta la tabla 1 aplicando la fórmula para la localidad de Orduña. A pesar de que las condiciones climáticas del área de intervención son extremas, llama la atención la gran diferencia de valores obtenidos entre las propuestas, de hasta $6,5^{\circ} \mathrm{C}$ de temperatura.

Como segunda consideración, es importante mencionar que también son muy escasas las investigaciones que indagan sobre las condiciones de confort en edificios tradicionales sin climatización artificial en climas de frío extremo. Uno de los pocos estudios realizados en climas fríos de altura fue desarrollado por Rijal y Yoshida (2006), quienes lograron identificar la temperatura neutra en viviendas del poblado de Lo Manthang, en el Distrito de Mustang en Nepal, ubicado a 3705 m s.n.m. bajo condiciones de temperaturas promedio en verano e invierno de 14 y $-1,3{ }^{\circ} \mathrm{C}$ respectivamente. Las temperaturas neutras durante el invierno identificadas por los pobladores en tres viviendas estudiadas fueron de $8,4^{\circ} \mathrm{C}, 10,6^{\circ} \mathrm{C}$ y $12,9^{\circ} \mathrm{C}$; el promedio fue de $10,7^{\circ} \mathrm{C}$.

Un segundo estudio, realizado por Mino-Rodríguez, Korolija y Altamirano (2018) en tres localidades cercanas a la ciudad de Quito, entre los 2300 y los 3100 m s.n.m., demuestra cómo la temperatura neutra desciende de manera evidente, junto con la propia temperatura media exterior, en la medida que se sube en altitud. A pesar de que las condiciones no son tan extremas como las de Orduña, la investigación permite reconocer las limitaciones de las fórmulas presentadas inicialmente en ámbitos tan particulares.

Tomando en cuenta los resultados del confort adaptativo, los estudios específicos realizados en climas fríos de altura, y a partir de las valoraciones subjetivas de los pobladores de la comunidad de Orduña expresadas en las actividades e intervenciones que se hicieron con anterioridad a la segunda etapa del prototipo, se estableció como meta alcanzar un valor de temperatura interior mínimo de entre 8 y $10^{\circ} \mathrm{C}$ en los momentos más fríos de la madrugada.

\section{Metodología}

Reconocidas las particularidades geográficas, climáticas y socioculturales de la zona, consideradas las técnicas y formas constructivas tradicionales y definidos los límites de confort, se desarrolla a continuación la metodología que se utilizó para implementar las estrategias bioclimáticas de diseño y monitorear térmicamente el prototipo de vivienda. Cabe mencionar que luego de la construcción del prototipo en 2015 y tras una etapa de monitoreo térmico de seis meses durante los meses más fríos del 2016, se intervino en una segunda ocasión, en el año 2017, para lograr la mejora del desempeño térmico del mismo.

\begin{tabular}{|c|c|c|c|c|}
\hline \multicolumn{5}{|c|}{ Rangos de temperaturas de confort según autores } \\
\hline \multirow{2}{*}{ Autores } & \multirow{2}{*}{ Fórmula } & \multicolumn{3}{|c|}{ Temperatura } \\
\hline & & media exterior (To) & de confort (Tc) & Rango \\
\hline $\begin{array}{l}\text { Nicol y Humphreys } \\
1998\end{array}$ & $T_{c}=11,9+0,534 x T_{o}$ & \multirow{3}{*}{$1,93^{\circ} \mathrm{C}$} & $12,93{ }^{\circ} \mathrm{C}$ & $09,48 / 16,38^{\circ} \mathrm{C}$ \\
\hline $\begin{array}{l}\text { DeDear y Brager } \\
1998\end{array}$ & $T_{c}=18,9+0,255 x T_{o}$ & & $19,39^{\circ} \mathrm{C}$ & $15,94 / 22,84^{\circ} \mathrm{C}$ \\
\hline $\begin{array}{l}\text { Nicol y Humphreys } \\
2002\end{array}$ & $T_{c}=13,5+0,540 \times T_{0}$ & & $14,54^{\circ} \mathrm{C}$ & $11,09 / 17,99^{\circ} \mathrm{C}$ \\
\hline
\end{tabular}

Tabla 1: Ecuaciones según los autores indicados y valores de temperatura media exterior, temperatura de confort y rango de temperaturas aceptables para el $80 \%$ de usuarios

Fuente: Elaboración propia (2020) 


\subsection{Las estrategias bioclimáticas y la construcción del prototipo (primera etapa)}

Partiendo del entendimiento del clima local, de las formas de habitar tradicionales y de las posibilidades y expectativas de los pobladores, se diseñó y construyó el prototipo original de vivienda, en 2015, en la localidad de Orduña. Fruto del diseño participativo con la comunidad, como punto de partida se decidió mantener la disposición típica de las viviendas de la región, la cual consta de un conjunto de volúmenes separados y organizados alrededor de un patio. Las condicionantes, como ya se ha adelantado, fueron principalmente la aceptación y apropiación del proyecto por parte de la comunidad, la búsqueda del confort térmico a través de un diseño bioclimático, sismorresistente y con la utilización preferente de materiales naturales y locales.

Con relación a las estrategias generales bioclimáticas consideradas en el diseño del prototipo, estas están alineadas con lo que proponen autores clásicos como Olgyay (1963) o Givoni (1998), y autores más recientes, igualmente renombrados, como Szokolay (2014) o Lechner (2014). En todos los casos se coincide en la necesidad de aislamiento de la envolvente, hermeticidad y ganancia solar como las tres estrategias fundamentales para un clima frío genérico. Las estrategias específicas consideradas en el prototipo han sido compiladas en un manual técnico de procesos constructivos disponible en línea (Rodríguez Larraín et al., 2016) y se resumen a continuación:

- Cimientos y sobrecimientos de piedra que dan estabilidad a los muros y los protegen de la humedad del suelo.

- Piso antihumedad con aislamiento y acabado de madera. Se disponen piedras de mayor a menor tamaño para evitar que la humedad del suelo suba por capilaridad y se coloca encima tierra alivianada: mezcla ligera de baja conductividad térmica compuesta por tierra y fibras naturales; densidad entre 1000 y $1200 \mathrm{~kg} / \mathrm{m}^{3}$. El acabado final es un entablado de madera, apoyado sobre durmientes, y los vacíos dejados entre estos son rellenados con lana de oveja.

- Muros de adobe de gran inercia térmica y de mayor aislamiento que la piedra (Figura 5), con adición de fibras de totora como elemento aislante natural. La totora, obtenida del Lago Titicaca, es una fibra natural de gran aislamiento térmico con la que se confeccionan unos tejidos de aproximadamente $4 \mathrm{~cm}$ de espesor, llamados 'qesanas'. Estudios previos sobre la conductividad térmica de la totora han demostrado su gran capacidad de aislamiento térmico (Ninaquispe, Weeks y Huelman, 2012; Hidalgo y García, 2018; Wieser, Onnis y Meli, 2018). A esto se le suma su gran disponibilidad en la región circundante al lago Titicaca. Se aprovechó el sistema sismorresistente del tejido de soguillas de nylon (drizas) y la viga collar de madera para unir la capa de aislamiento de totora al muro. Adobes y totora son finalmente recubiertos por un revestimiento de tierra alivianada con pajas de gramíneas y un enlucido final de tierra y arena fina.

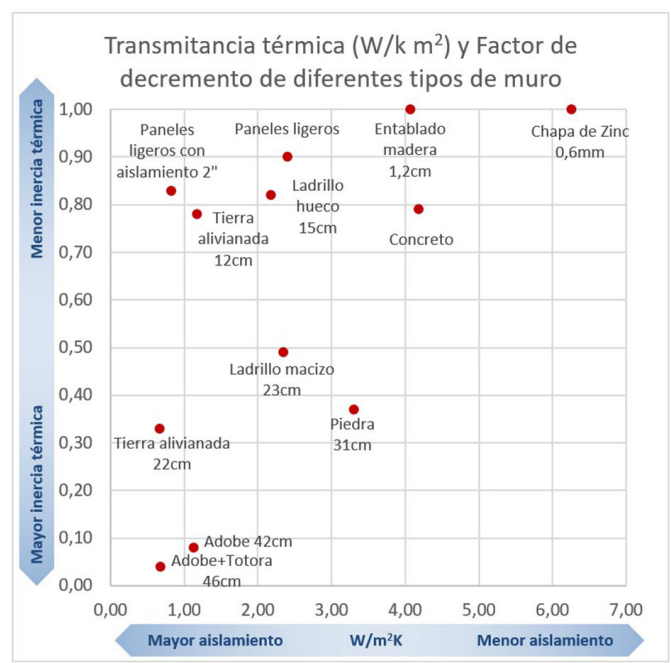

Figura 5: Gráfico de transmitancias térmicas (capacidad de aislamiento) y factor de decremento (capacidad de amortiguamiento) de diversos cerramientos de muro Fuente: Elaboración propia (2020)

- $\quad$ Techo compuesto por una estructura de viguetas de madera sobre la que se apoyan 'qesanas' de totora. Enlucido de yeso bajo las 'qesanas' y una capa de tierra alivianada por encima de ellas como aislamiento adicional y protección de la totora. Se coloca finalmente una plancha ondulada metálica hacia el exterior con el fin de proteger e impermeabilizar los materiales naturales inferiores.

- Vanos de ventana de dimensiones reducidas con marcos de madera, herméticos y con contraventanas de madera contraplacada y aislamiento de lana de oveja al interior. Puertas con similar aislamiento y cierre hermético.

- Claraboya en el techo del vestíbulo que permite e ingreso a los dormitorios para la captación solar directa. Considerando la gran verticalidad de recorrido solar en el Perú durante todo el año, se aprovechan de esta manera las horas de mayor radiación, cercanas al mediodía.

Una vez decididos los materiales y componentes a utilizar para lograr el aislamiento, la hermeticidad y la ganancia solar del prototipo, se utilizó el software Design Builder para simular el comportamiento térmico dinámico y tomar las decisiones finales, principalmente en torno al espesor de los elementos aislantes de la envolvente y a las dimensiones de la claraboya para la captación solar en el vestíbulo. Establecido como objetivo el elevar las temperaturas interiores mínimas alrededor de los $10^{\circ} \mathrm{C}$ en las horas más frías del día, se determinó que resultaba suficiente la incorporación de una sola 'qesana' de totora (espesor aproximado de $4 \mathrm{~cm}$ ) y que la claraboya cuente con un área aproximada de $1.20 \mathrm{~m}^{2}$.

El volumen principal resultante, que contiene las habitaciones y el vestíbulo, tiene unas medidas exteriores de 10.54 x 4.00 m (área ocupada de $42.16 \mathrm{~m}^{2}$ ) y los dos dormitorios unas medidas interiores de $3.00 x$ $3.20 \mathrm{~m}$ (9.60 $\mathrm{m}^{2}$ cada uno); la altura libre interior oscila entre 2.10 y $2.50 \mathrm{~m}$. Se muestran a continuación tanto un esquema que resume el funcionamiento de las 
estrategias bioclimáticas mencionadas, así como algunas fotografías de la propuesta construida (Figuras 6 y 7 ).

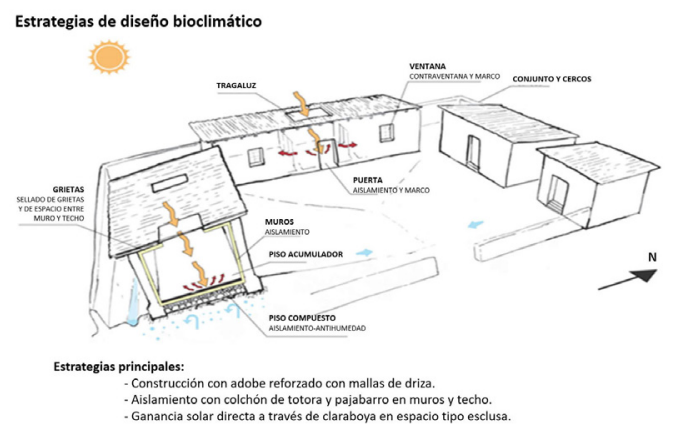

Figura 6: Esquema de la propuesta con las estrategias bioclimáticas pasivas

Fuente: Centro Tierra, PUCP (2017)

Como ya se adelantó, a pesar de las simulaciones realizadas, los resultados térmicos del prototipo en su primera etapa fueron prometedores, pero no del todo satisfactorios. La temperatura interior se mantuvo en $5^{\circ} \mathrm{C}$ en los momentos más fríos de la madrugada; 8 a $9^{\circ} \mathrm{C}$ por encima de la temperatura exterior y alrededor de $5{ }^{\circ} \mathrm{C}$ por encima de una habitación de una cabaña típica local. Se decidió entonces intervenir el edificio nuevamente, incorporando algunas mejoras detectadas a partir de lo observado y de los resultados obtenidos.
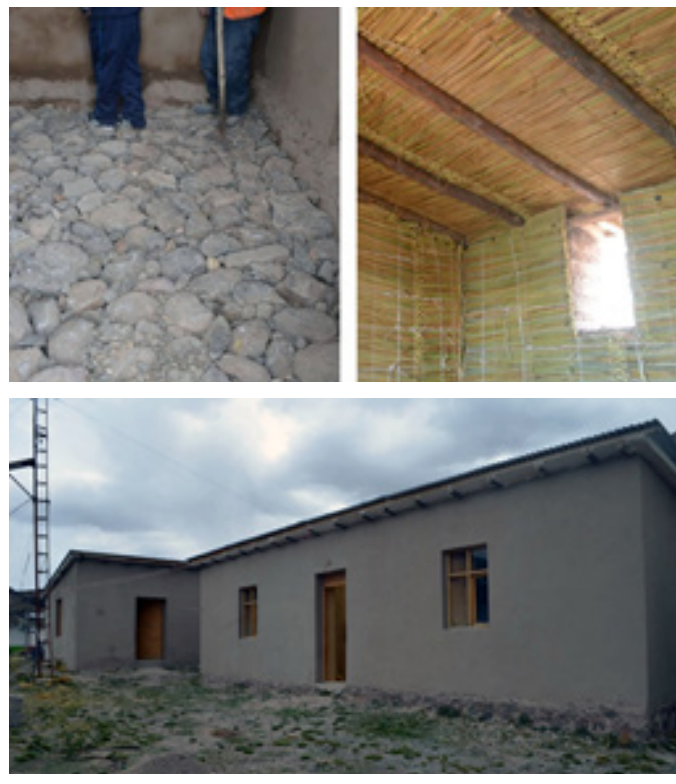

Figura 7: Fotos del prototipo: Detalles del piso antihumedad (arriba izquierda), muro de adobe con capa de totora, ambas confinadas con el sistema antisísmico de drizas (arriba derecha) y prototipo terminado (abajo). Fuente: Centro Tierra, PUCP (2018)

\subsection{Mejoras introducidas (segunda etapa)}

A partir de los resultados y la evaluación del desempeño térmico alcanzado por el prototipo, se plantearon cuatro propuestas específicas para implementar en una segunda intervención: (1) conseguir una mayor hermeticidad en los vanos, (2) elevar el nivel de aislamiento en el techo, (3)
(3) ampliar el área traslúcida de la claraboya para aumentar la ganancia solar directa y (4) asegurar el uso correcto de los cerramientos asociados al sistema de captación solar.

Como se puede advertir, las estrategias corresponden a aquellas aplicadas en la primera etapa; lo que se plantean son ajustes y una condición más radical de las mismas. Se desarrolla a continuación el detalle de las cuatro propuestas de mejora mencionadas y el protocolo de medición seguido para la obtención de datos. Dicha intervención se realizó en septiembre de 2017, mientras que el monitoreo térmico se llevó a cabo en septiembre de 2018.

La primera mejora fue la ampliación de la claraboya, a la que se le asignaron unas dimensiones finales que prácticamente coincidieron con el área del vestíbulo (Figura 8, arriba). El resultado fue la creación de un invernadero central que casi quintuplicó el área original del material traslúcido, buscando el máximo aprovechamiento de la radiación solar en las horas cercanas al mediodía.

Una segunda mejora implicó la colocación de aislamiento adicional en el techo (Figura 8, abajo). Manteniendo la lógica de la capa de totora como aislante, con el recubrimiento de yeso en la parte interior de la habitación, se añadió una capa adicional de tierra alivianada con trozos de totora de $4 \mathrm{~cm}$ de espesor y de $600 \mathrm{Kg} / \mathrm{m} 3$ de densidad por encima de las 'qesanas' de totora. Finalmente, se superpuso nuevamente una plancha ondulada metálica para proteger los elementos inferiores de la lluvia, el sol y el viento.
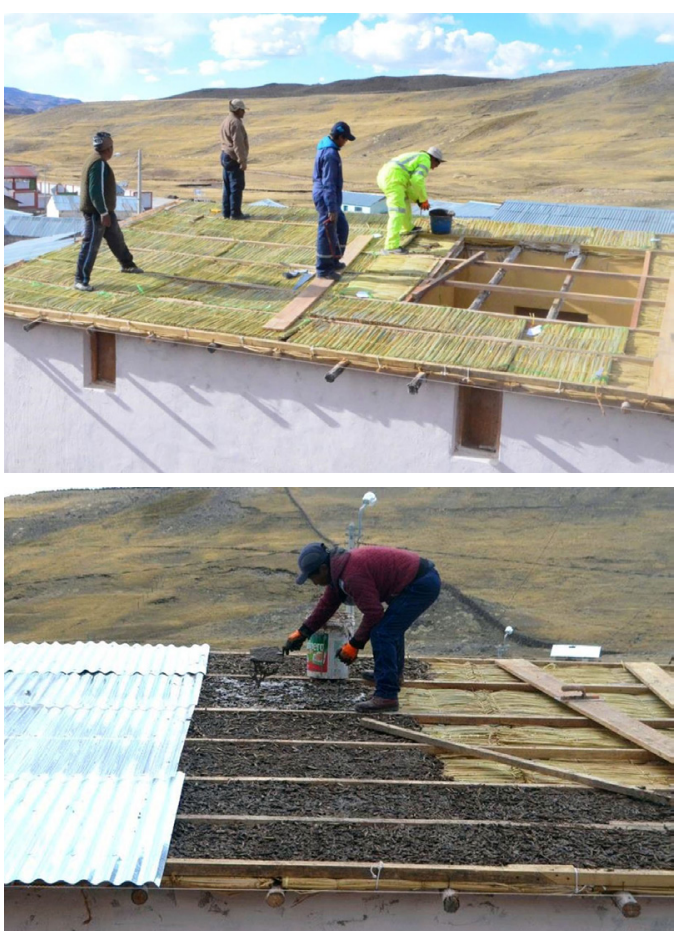

Figura 8: Detalle de la ampliación de la claraboya del vestíbulo-invernadero (arriba) y adición de capa adicional de tierra alivianada y plancha ondulada impermeable en la cubierta del prototipo

Fuente: Centro Tierra, PUCP (2018) 
Para valorar mejor el aumento de la capacidad aislante de los muros y de la cubierta del prototipo, en sus dos etapas de intervención, y retomando algunos datos expuestos en la Figura 5, se presentan a continuación los valores de transmitancia térmica de dichos elementos, comparándolos con otras soluciones tradicionales y contemporáneas locales (Tabla 2)

\begin{tabular}{|c|c|}
\hline \multicolumn{2}{|c|}{ Valores de transmitancia térmica } \\
\hline Muros & $\begin{array}{l}\text { Transmitancia } \\
\text { térmica, } \mathrm{W} / \mathrm{m}^{2}{ }^{\circ} \mathrm{C}\end{array}$ \\
\hline \multicolumn{2}{|c|}{ Soluciones típicas } \\
\hline Piedra $(30 \mathrm{~cm})$ & 3,221 \\
\hline Adobe $(42 \mathrm{~cm})$ & 1,088 \\
\hline \multicolumn{2}{|c|}{ Prototipo $1^{\mathrm{a}}$ y $2^{\mathrm{a}}$ etapa } \\
\hline Adobe + totora $(42+4 \mathrm{~cm})$ & 0,667 \\
\hline Cubiertas & $\begin{array}{c}\text { Transmitancia } \\
\text { térmica, } \mathrm{W} / \mathrm{m} 2{ }^{\circ} \mathrm{C}\end{array}$ \\
\hline \multicolumn{2}{|c|}{ Soluciones típicas } \\
\hline Tejido de Ichu (6 cm) & 1,155 \\
\hline Plancha metálica (0,03 mm) & 4,000 \\
\hline \multicolumn{2}{|c|}{ Prototipo 1 a etapa } \\
\hline $\begin{array}{l}\text { Yeso + totora + tierra + plan- } \\
\text { cha metálica }(2+4+1+0 \mathrm{~cm})\end{array}$ & 1,325 \\
\hline \multicolumn{2}{|c|}{ Prototipo 2 a etapa } \\
\hline $\begin{array}{l}\text { Yeso + totora + tierra ali- } \\
\text { vianada }+ \text { plancha metálica } \\
(2+4+4+0 \mathrm{~cm})\end{array}$ & 0,916 \\
\hline
\end{tabular}

Tabla 2 Valores de transmitancia térmica de diferentes soluciones de muros y cubiertas

Fuente: Elaboración propia (2020)

La tercera mejora buscó asegurar un mínimo de hermeticidad en los ambientes y se realizó específicamente en los vanos de puertas y ventanas. Las condiciones rigurosas del clima, con grandes cambios de temperaturas entre el día y la noche, la radiación solar extrema y la presencia estacional de precipitaciones, generaron dilataciones en la madera de los vanos e hizo que los encuentros entre los marcos y las hojas, así como con los vidrios de las ventanas, perdieran precisión. Esta situación permitió el ingreso excesivo de infiltraciones indeseadas que fueron controladas interviniendo la carpintería con trabajos de mayor rigurosidad y con la inserción de tapajuntas, cerrojos y cortinas gruesas interiores en todas las ventanas.

Una última mejora se relacionó al uso mismo de sistema de captación, buscando asegurar el correcto funcionamiento de este a partir de la manipulación adecuada de las aberturas. Se identificó en las mediciones de la primera etapa la recurrencia en el hábito de dejar la puerta exterior abierta durante el día; esta condición resulta comprensible cuando la temperatura del aire exterior cerca al mediodía suele ser mayor que la de interior de una cabaña tradicional. Pero la propuesta con el invernadero no es compatible con esta condición. Resulta imprescindible mantener la puerta exterior cerrada durante todo el tiempo, mientras que las puertas hacia los dormitorios deberían necesariamente quedar abiertas durante el día y cerradas en la noche. Esta condición permite que el aire caliente del vestíbulo/ invernadero ingrese por convección a los dormitorios y no se pierda hacia el exterior. Para asegurar la realización de las mediciones específicas con la correcta manipulación de los cerramientos, el prototipo fue habitado por un miembro del equipo durante una semana continua en la época más fría del año.

\subsection{Las mediciones térmicas}

Se registraron las condiciones exteriores de forma continua, específicamente la temperatura del aire, la humedad relativa del aire, la radiación solar y la dirección, velocidad y frecuencia de los vientos. Al interior del prototipo se midieron la temperatura $y$ la humedad relativa del aire en los tres ambientes. Se registraron adicionalmente diversas situaciones interiores y exteriores con una cámara termográfica para identificar la temperatura superficial de la envolvente y localizar los eventuales puentes térmicos del edificio. Por último, se obtuvieron datos de concentración de dióxido de carbono del aire (partes por millón, ppm) para comprobar que el nivel de hermeticidad no ponga en riesgo la salubridad del ambiente interior

A continuación, se muestra la lista de los instrumentos de medición utilizados en el monitoreo térmico de prototipo, así como los datos registrados, sus unidades y los intervalos programados para dicho fin (ver Tabla 3).

\begin{tabular}{|c|c|c|}
\hline $\begin{array}{c}\text { Instrumento } \\
\text { medición }\end{array}$ & $\begin{array}{l}\text { Datos registrados } \\
\text { (unidades) }\end{array}$ & Observaciones \\
\hline $\begin{array}{l}\text { Estación meteoro- } \\
\text { lógica } \\
\text { Marca: DAVIS } \\
\text { Modelo: Vantage } \\
\text { Pro } 2 \text { Plus }\end{array}$ & $\begin{array}{l}\text { Temperatura del } \\
\text { aire }\left({ }^{\circ} \mathrm{C}\right) \\
\text { Humedad relativa del } \\
\text { aire }(\%) \\
\text { Radiación solar, (W/ } \\
\mathrm{m}^{2} \text { ) } \\
\text { Vientos (dirección, } \\
\mathrm{m} / \mathrm{s}, \%) \\
\text { Lluvias (mm) }\end{array}$ & $\begin{array}{l}\text { Instrumento que } \\
\text { registra las condi- } \\
\text { ciones exteriores } \\
\text { con intervalos de } \\
1 \text { hora. }\end{array}$ \\
\hline $\begin{array}{l}\text { Registrador de } \\
\text { datos } \\
\text { Marca: ONSET } \\
\text { Modelo: Hobo H08- } \\
\text { 003-02 }\end{array}$ & $\begin{array}{l}\text { Temperatura del } \\
\text { aire }\left({ }^{\circ} \mathrm{C}\right) \\
\text { Humedad relativa del } \\
\text { aire }(\%)\end{array}$ & $\begin{array}{l}\text { Instrumentos (03) } \\
\text { que registran las } \\
\text { condiciones de } \\
\text { las habitaciones } \\
\text { interiores del } \\
\text { prototipo con } \\
\text { intervalos de } 1 \\
\text { hora. }\end{array}$ \\
\hline $\begin{array}{l}\text { Registrador de } \\
\text { datos } \\
\text { Marca: SPER } \\
\text { SCIENTIFIC } \\
\text { Modelo: } 800050\end{array}$ & $\begin{array}{l}\text { Concentración de } \\
\text { CO2 (ppm) }\end{array}$ & $\begin{array}{l}\text { Instrumento que } \\
\text { mide la concen- } \\
\text { tración de dióxido } \\
\text { de carbono en } \\
\text { tres momentos } \\
\text { del día: antes de } \\
\text { dormir ( } 10 \mathrm{pm}) \text {, a } \\
\text { la hora de desper- } \\
\text { tarse ( } 6 \text { am) y en } \\
\text { la tarde }(6 \mathrm{pm}) \text {. }\end{array}$ \\
\hline $\begin{array}{l}\text { Cámara termo- } \\
\text { gráfica } \\
\text { Marca: FLIR } \\
\text { Modelo: E5 }\end{array}$ & $\begin{array}{l}\text { Temperatura superfi- } \\
\text { cial }\left({ }^{\circ} \mathrm{C}\right)\end{array}$ & $\begin{array}{l}\text { Instrumento de } \\
\text { registro gráfico de } \\
\text { las temperaturas } \\
\text { superficiales des- } \\
\text { de una posición } \\
\text { determinada. }\end{array}$ \\
\hline
\end{tabular}

Tabla 3: Instrumentos de medición utilizados en el monitoreo del prototipo

Fuente: Elaboración propia (2020) 
Los resultados de las mediciones exteriores permitieron confirmar las características climáticas típicas del emplazamiento, además de ratificar la condición de clima marcadamente constante y predecible que ya se había registrado en las mediciones previas. De otro lado, los valores obtenidos durante la semana de mediciones interiores, con la presencia de personas y la manipulación apropiada de los cerramientos en vanos, permitieron identificar no solo el desempeño térmico, sino también la calidad del aire al interior del prototipo.

\section{Resultados y discusión}

Se presentan a continuación los resultados de las mediciones de las temperaturas exterior e interiores, tanto de la primera etapa del proyecto como de la segunda (Figura 9). En la primera, las mediciones fueron realizadas durante todo el mes de agosto de 2015, mostrándose en el gráfico un día típico obtenido a partir del promedio mensual; como se adelantó, en esa oportunidad no se aseguró la ocupación permanente ni los protocolos de uso de las aberturas de los vanos. En el segundo cuadro se muestran los resultados de las mediciones que se realizaron después de la segunda intervención, cuyos valores representan las temperaturas promedio de cuatro días de medición en septiembre de 2018. Se resalta la gran similitud entre las condiciones exteriores de ambos momentos del año, situación que es predecible en esta región altiplánica.
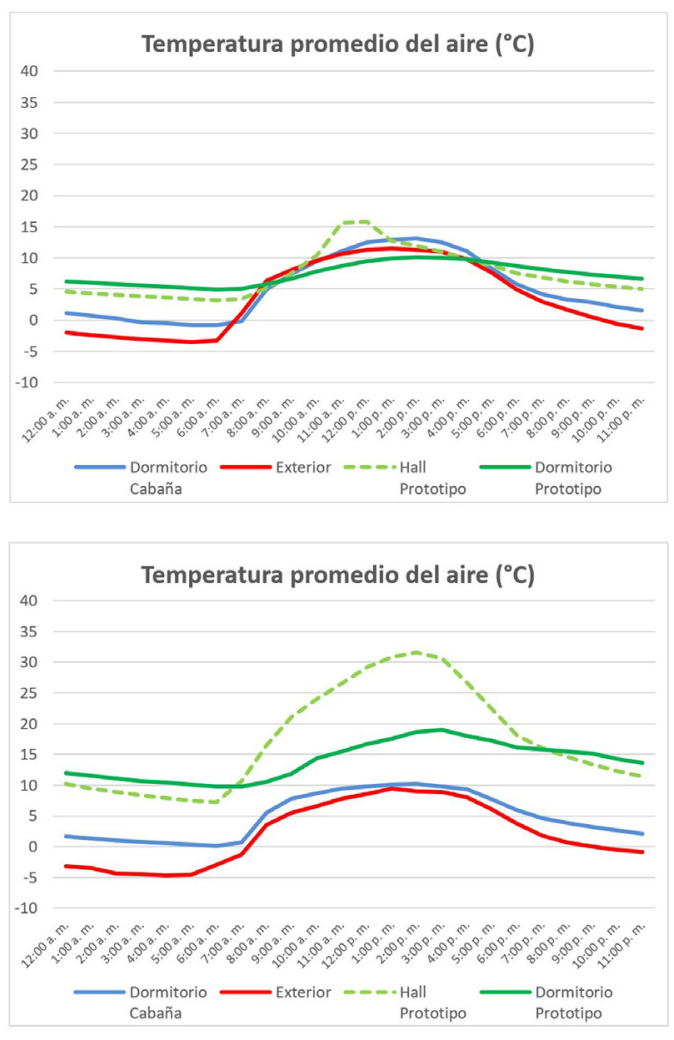

Figura 9: Gráficos con valores de temperatura interior del prototipo de la primera etapa en 2015 (arriba) y de la segunda etapa en 2018 (abajo) con las mejoras implementadas

Fuente: Elaboración propia (2020)
La comparación de los valores obtenidos en ambas mediciones evidencia una mejora significativa en el desempeño térmico del prototipo a partir de la segunda intervención. Con las propuestas implementadas, la temperatura interior se eleva $5{ }^{\circ} \mathrm{C}$ adicionales en relación con la primera etapa y, lo que es más importante, alcanza valores mínimos de $10{ }^{\circ} \mathrm{C}$ en los momentos más fríos, prácticamente $15{ }^{\circ} \mathrm{C}$ por encima de las condiciones exteriores.

En relación al nivel de hermeticidad de los dormitorios, se realizaron mediciones de concentración de dióxido de carbono (CO2, ppm) durante dos días en los que el equipo de investigación ocupó las habitaciones de manera intensiva, en tres diferentes horas del día: al momento de ocupar el espacio después de encontrarse desocupado todo el día (alrededor de las 18:00 horas), antes de dormir (alrededor de las 22:00 horas) y al momento de despertarse (alrededor de las 06:00 horas), luego de permanecer dos personas durmiendo toda la noche. Esta última condición se preveía como la más delicada y, como era de esperarse, en ella se presentó la mayor concentración de CO2 (Tabla 4). Si bien es cierto que los resultados están dentro de valores considerados como aceptables, al no pasar el umbral de los 1200 ppm (ASHRAE, 2016). Los resultados también indican que se encuentran al límite y que no resulta prudente aumentar más la hermeticidad sin considerar alguna estrategia especial de ingreso de aire precalentado.

\begin{tabular}{|c|c|c|c|}
\hline Ambiente & $\begin{array}{c}\mathbf{1 8 : 0 0} \\
\text { horas }\end{array}$ & $\begin{array}{c}\mathbf{2 2 : 0 0} \\
\text { horas }\end{array}$ & $\begin{array}{c}\mathbf{0 6 : 0 0} \\
\text { horas }\end{array}$ \\
\hline Dormitorio 1 & 628 & 523 & 982 \\
\hline Dormitorio 2 & 653 & 544 & 1143 \\
\hline
\end{tabular}

Tabla 4: Niveles de concentración de dióxido de carbono, en partes por millón (CO2, ppm)

Fuente: Elaboración propia (2020)

Para lograr identificar y localizar posibles puentes térmicos, se utilizó una cámara termográfica a diferentes horas del día, tanto desde el interior como del exterior del prototipo. Un momento clave del día, en el que el interior mantiene una temperatura moderada mientras que al exterior se presentan los valores mínimos, se da en las primeras horas de la mañana. La imagen presentada a continuación (Figura 10), que coincide con dicho momento, evidencia con cierta claridad la forma en la que el calor sale del prototipo.

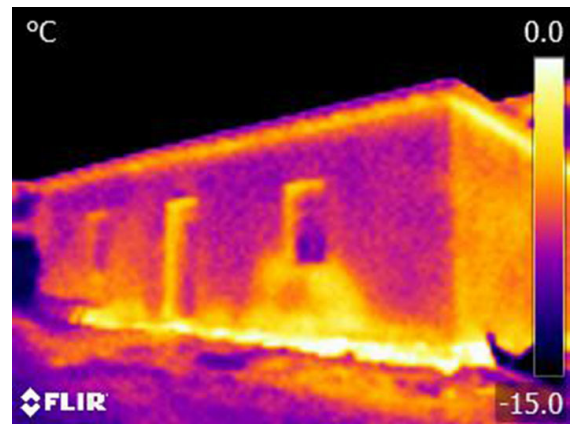

Figura 10: Fotografía tomada con cámara termográfica a las 06:00 horas, en la que se revelan los puentes térmicos del prototipo

Fuente: Centro Tierra, PUCP (2020) 
Un primer puente térmico se presenta en el perímetro de los vanos de la puerta y de las ventanas; la infiltración casi imperceptible y una contraventana con un aislamiento que resulta inevitablemente menor que la que posee el muro de adobe y totora, hace que el calor escape con mayor facilidad que en el resto del muro. Un segundo puente térmico, más evidente aún, es aquel que se presenta en el perímetro del prototipo en contacto con el suelo, que coincide a su vez con la piedra utilizada en el cimiento y sobrecimiento. A pesar de que se ha asegurado una continuidad de la totora como elemento aislante de muro y de la tierra alivianada junto con la madera como elemento aislante del piso interior, resulta evidente e problema que significa la mayor conductividad térmica de la piedra frente a la del adobe.

\section{Conclusiones}

Los resultados de las mediciones al interior del prototipo, específicamente aquellos obtenidos a partir de la segunda intervención, logran demostrar que es posible obtener condiciones de confort en las viviendas ubicadas en regiones extremadamente frías del altiplano peruano, a partir de la concepción de estrategias pasivas y de la utilización casi exclusiva de materiales locales y naturales. El aprovechamiento de la radiación solar, acompañada del aislamiento de la envolvente, de la hermeticidad de conjunto y del correcto uso de los vanos para contener el calor ganado, fueron las principales estrategias bioclimáticas que permitieron lograr dicho objetivo.

Es importante reconocer que la posibilidad de obtener confort térmico en condiciones de clima de frío extremo sin el uso de sistemas artificiales no es algo que se presupone en la bibliografía clásica de arquitectura bioclimática; esto se puede identificar incluso en las estrategias sugeridas por Givoni que se muestran en la Figura 4, en la que se indica el uso necesario de calefacción. En el caso particular del clima frío tropical de altura, se cuentan con dos características climáticas particulares que, aprovechándolas de forma correcta, posibilitan prescindir del gasto energético que supone el uso de calefacción. Si bien las temperaturas durante la noche caen por debajo de $0{ }^{\circ} \mathrm{C}$, durante el día estas suben alrededor de los $12^{\circ} \mathrm{C}$; pero lo más importante es reconocer que la cercanía a la Línea Ecuatorial determina una gran verticalidad en el recorrido solar, poca diferencia estacional y cierta similitud en la duración entre el día y la noche a lo largo del año. Si a esto se le suma la menor nubosidad que se presenta en los meses más fríos, el uso potencial de la intensa radiación solar se convierte en la estrategia bioclimática clave.

Las eventuales mejoras que se presentan como posibles propuestas a futuro, manteniendo las exigencias de soluciones pasivas y naturales, pasan principalmente por la necesidad de reducir el puente térmico que existe entre el muro con aislamiento y el cimiento de piedra, y por la posibilidad de hermetizar aún más la envolvente, pero precalentando el ingreso de un flujo de aire, ya sea a través de intercambiadores de calor o por ventilación subterránea. En la medida que se decida la incorporación de estrategias bioclimáticas activas, reconociendo que resultan más costosas en su implementación y mantenimiento, estas se implementarían principalmente con la incorporación de sistemas de calentamiento solar y almacenaje de fluidos (agua o aire) durante el día, e irradiando el calor contenido durante la noche.

Por último, se reconoce la necesidad de un estudio más sistemático y de ámbito regional, que permita definir con mayor precisión y objetividad los rangos de confort térmico para una zona de características climáticas tan especiales y rigurosas.

\section{Agradecimientos}

La presente investigación ha sido posible gracias al financiamiento recibido del Consejo Nacional de Ciencia, Tecnología e Innovación Tecnológica (CONCYTEC) y de la Pontificia Universidad Católica del Perú (PUCP), así como a la participación de la comunidad de Orduña y del Grupo de Investigación Centro Tierra, PUCP.

Cómo citar este artículo/How to cite this article: Martín Wieser, M., Rodríguez-Larraín, S. y Onnis, S. (2021). Estrategias bioclimáticas para clima frío tropical de altura. Validación de prototipo en Orduña, Puno, Perú. Estoa. Revista de la Facultad de Arquitectura y Urbanismo de la Universidad de Cuenca, 10(19), 9-19. doi: 10.18537/est.v010. n019.a01 


\section{Referencias bibliográficas}

ASHRAE, A. (2016). ASHRAE Standard 62.1-2016. Ventilation for acceptable indoor air quality. American Society of Heating, Refrigerating, and Air-Conditioning Engineers, Inc

De Dear, R. J. y Brager, G. S. (1998). Developing an adaptive model of thermal comfort and preference. Proceedings of the 1998 ASHRAE Winter Meeting (pp. 145-167). 104(Pt 1A), ASHRAE.

Givoni, B., (1998). Climate Considerations in Building and Urban Design John Wiley \& Sons.

Hidalgo-Cordero, J. F. y García-Navarro, J. (2018). Totora (Schoenoplectus californicus (CA Mey.) Soják) and its potential as a construction material. Industrial Crops and Products, 112, 467-480.

Jiménez, G., Wieser, M. y Biondi, S. (2017). Improving Thermal Performance of Traditional Cabins in the HighAltitude Peruvian Andean Region. PLEA Conference (pp. 4101-4108). Network for Comfort and Energy Use in Buildings.

Lechner, N. (2014). Heating, cooling, lighting: Sustainable design methods for architects. John Wiley \& Sons.

Marsh, A. (2019). Psychrometric Chart. Software en línea. http://andrewmarsh.com/software/psychro-chart-web/

Mino-Rodriguez, I., Korolija, I. y Altamirano, H. (2018). Thermal comfort in dwellings in the subtropical highlands Case study in the Ecuadorian Andes. Conference: 10th Windsor Conference - Rethinking Comfort (pp. 760 774). NCEUB - Network for Comfort and Energy Use in Buildings.

Nicol, J. F. y Humphreys, M. (1998). Understanding the adaptive approach to thermal comfort. ASHRAE transactions, 104(1), 991-1004.

Nicol, J. F. y Humphreys, M. A. (2002). Adaptive therma comfort and sustainable thermal standards for buildings. Energy and buildings, 34(6), 563-572.

Ninaquispe Romero, L., Weeks, S. y Huelman, P. (2012). Totora: A Sustainable Insulation Material for the Andean Parts of Peru. In: Proceedings - 28th International PLEA Conference.

Olgyay, V. (1963). Design with climate: Bioclimatic approach to architectural regionalism. Princeton University Press.

Onnis, S., Rodríguez-Larraín, S., Meli, G. y Loayza León, L. (2018). Modelo de transferencia tecnológica para la vivienda altoandina. Memorias del $18^{\circ}$ SIACOT (pp. 606615). Proterra

Pulgar Vidal, J. (1996). Geografía del Perú. Las ocho regiones naturales. La Regionalización Transversal. La Sabiduría Ecológica Tradicional. PEISA.
Rijal, H. B. y Yoshida, H. (2006, April). Winter thermal comfort of residents in the Himalaya region of Nepal. In Proceeding of international conference on comfort and energy use in buildings-getting them right (Windsor) (pp. 1-15). NCEUB-Network for Comfort and Energy Use in Buildings.

Rodríguez-Larraín, S., Onnis, S. y Vargas-Neumann, J. (2016). Transferencia tecnológica para la vivienda altoandina. In: Proceedings - TERRA 2016 XIIth World Congress on Earthen Architecture (pp. 339-344). CRAterre.

Rodríguez-Larraín, S., Alvarino, M., Onnis, S., Wieser, M., Jiménez, C., Meli, G., Vargas-Neumann, J. y Sosa, C. (2016). Manual del promotor técnico para la construcción de la vivienda altoandina segura y saludable. Centro Tierra, CIAC, PUCP. https://issuu.com/centrotierra/ docs/1. manual_del_promotor_t_cnico

Szokolay, S. V. (2014). Introduction to architectural science: the basis of sustainable design. Routledge.

Wieser, M., Onnis, S. y Meli, G. (2018). Conductividad Térmica de la tierra alivianada con fibras naturales en paneles de quincha. Proceedings of the SIACOT (pp.199208). Proterra 